\title{
Editorial
}

\section{Bid Kumar}

Following on another very successful scientific meeting at Old Trafford Manchester, we usher you back to the reading desk. This first issue of Ultrasound in 2019 comes with the most number of pages since the publication of Ultrasound began. I thank our publishers for this allowance. Here, we enclose a pictorial assay and six works of original research, besides the usual section on echoes written by Hazel Edwards.

Chambers and colleagues presented an essay enriched with images of children's musculoskeletal injuries. They emphasized the utility of ultrasound in such cases. The images in this article add vividity to their description.

Orr and colleagues investigated the impact of a new ultrasound curriculum, incorporating transabdominal simulators within the first year of training in a UK radiology academy. They reported the result of their experience with a group of trainees who underwent training with the help of simulators. Their findings lend support to this technique of training which is gaining popularity although practical implementation has implication on resources, particularly financial.

Flavell et al. investigated a standardized method that controls and records probe force, inclination and roll during ultrasound imaging of transversus abdominis. They also aimed to find out the utility and intraobserver reliability with this kind of standardization during ultrasound imaging of transversus abdominis thickness measurement in chronic low back pain patients. They conducted transversus abdominis imaging over two separate measurement sessions using a novel method to standardize probe parameters. Their findings are interesting and should lead to further research in this area.
Cochard's study about point of care ultrasound (POCUS) aimed to improve the hospital practitioners' knowledge and skills through a hands-on skills practicum. Their study demonstrated that a brief skills practicum significantly improves the hospitalists' confidence and knowledge regarding ultrasound image acquisition and interpretation in the short term. Long-term confidence and usage wane, which appears to be due to the lack of machine access.

Hamza et al. wanted to find out the concordance between radiologic and pathologic tumor size to decide about the accuracy of radiologic and clinical staging. They studied 406 cases and inform us that the prediction of exact surgical technique based upon radiological findings alone may be risky.

Johnson and colleagues investigated sonographer experiences of difficult news delivery training and preferences for training techniques and assessed whether news delivery training is associated with lower burnout and higher wellbeing. Their findings once again underlined the importance of training and skill acquisition.

Chua reviewed the adequacy and diagnostic accuracy of carrying out biopsy of sub-centric thyroid nodules. They biopsied 245 such thyroid nodules and their results indicated relatively low diagnostic accuracy and false-positive reports of such biopsy. However, their circumstances indicated that the results influenced the clinical management of that group of patients.

Once again, we request our readers and all other enthusiasts to send us their scientific work for consideration of publication in our journal which is constantly gaining in readership and popularity. We thank all our readers for their continuing support for Ultrasound.
Department of Obstetrics and Gynaecology, Wrexham Maelor Hospital, Wrexham, UK

Corresponding author:

Bid Kumar, Department of Obstetrics and Gynaecology, Wrexham Maelor Hospital, Wrexham LL13 7TD, UK.

Email: bidyut.kumarawales.nhs.uk/kumar.bidyutayahoo.co.uk 